\title{
NONTANGENTIAL LIMITS AND FATOU-TYPE THEOREMS ON POST-CRITICALLY FINITE SELF-SIMILAR SETS
}

\author{
RICARDO A. SÁENZ
}

\begin{abstract}
In this paper we study the boundary limit properties of harmonic functions on $\mathbb{R}_{+} \times K$, the solutions $u(t, x)$ to the Poisson equation

$$
\frac{\partial^{2} u}{\partial t^{2}}+\Delta u=0
$$

where $K$ is a p.c.f. set and $\Delta$ its Laplacian given by a regular harmonic structure. In particular, we prove the existence of nontangential limits of the corresponding Poisson integrals, and the analogous results of the classical Fatou theorems for bounded and nontangentially bounded harmonic functions.
\end{abstract}

\section{INTRODUCTION}

There has recently been a growing interest in the study of analysis on fractals, in particular post-critically finite (p.c.f.) self similar sets and their harmonic structure defined by Kigami Kig93. Analogous questions from classical analysis have been asked on the setting of p.c.f. fractals, from spectral theory of the Laplacian [ASST03, CSW09, ORS10, functional analysis [Str03, RS10, IR10] and differential equations DSV99, Str05, Pel07.

In this paper we study the boundary limit properties of harmonic functions on the tube $\mathbb{R}_{+} \times K$, the solutions $u(t, x)$ to the Poisson equation

$$
\frac{\partial^{2} u}{\partial t^{2}}+\Delta u=0
$$

where $K$ is a p.c.f. set and $\Delta$ its Laplacian given by a regular harmonic structure. In Section 3 we define the Poisson kernel and prove its elementary properties, as well as proving the existence of nontangential limits of Poisson integrals in the boundary $t \rightarrow 0$. Nontangential limits are defined in terms of proper "cones", depending on the Hausdorff dimension of $K$ with respect to effective resistence metric.

In Section 4 we prove an analogous Fatou theorem for bounded Dirichlet harmonic functions on $\mathbb{R}_{+} \times K$. We also extend these results to Dirichlet harmonic functions with uniformly bounded $L^{p}$ norms, $1 \leq p<\infty$.

We finish the paper with the analogous version to the local Fatou theorem, in Section 5. for nontangentially bounded harmonic functions on $\mathbb{R}_{+} \times K$. As we make use of estimates from below for the Neumann heat kernel on $K$, we prove this results only for nested fractals [Lin90].

Date: October 26, 2018.

2000 Mathematics Subject Classification. 28A80, 31B25.

Key words and phrases. Fractals, p.c.f. sets, Poisson integrals, boundary behavior of harmonic functions. 


\section{Preliminaries}

2.1. P.c.f. self-similar structures. Let $\left(K, S,\left\{F_{i}\right\}_{i \in S}\right)$ be a self-similar structure. $W_{m}=S^{m}$ is the set of words of length $m$, and $W_{*}=\bigcup_{m \geq 0} W_{m}$, where $W_{0}=$ $\{\emptyset\}$ and $\emptyset$ is called the empty word. For $w \in W_{m}$, we write $F_{w}=F_{w_{1}} \circ \cdots \circ F_{w_{m}}$ ( $F_{\emptyset}$ is set to be the identity mapping) and $K_{w}=F_{w}(K)$. The critical set of $K$ is the set

$$
\mathcal{C}=\bigcup_{\substack{i, j \in S \\ i \neq j}} K_{i} \cap K_{j}
$$

and the post-critical set is given by $V_{0}=\left\{p \in K: \exists w \in W_{*}, F_{w}(p) \in \mathcal{C}\right\}$. We say that $K$ is a post-critically finite (p.c.f.) self-similar structure if the set $V_{0}$ is finite. From now on, we will assume that $K$ is a p.c.f. self-similar structure, and that $V_{0} \neq \emptyset$.

$V_{0}$ will be called the boundary of $K$. We define $V_{m}=\bigcup_{w \in W_{m}} F_{w}\left(V_{0}\right)$, and $V_{*}=\bigcup_{m \geq 0} V_{m}$. Basic properties and examples of p.c.f. self-similar structures can be found in [Kig01, Section 1.3].

If $0<\mu_{i}<1$ and $\sum_{i \in S} \mu_{i}=1$, let $\mu$ be the Bernoulli measure with weights $\left(\mu_{i}\right)_{i \in S}$. It satisfies $\mu\left(K_{w}\right)=\mu_{w}=\mu_{w_{1}} \cdots \mu_{w_{m}}$ for $w \in W_{m}$ and, for any integrable function $f$ on $K$,

$$
\int_{K} f d \mu=\sum_{w \in W_{m}} \mu_{w} \int_{K} f \circ F_{w} d \mu .
$$

We define the Banach spaces $L^{p}(K, \mu), 1 \leq p \leq \infty$, as usual.

2.2. Harmonic structure. Let $(D, \mathbf{r})$ be a regular harmonic structure on $K$, where $\mathbf{r}=\left(r_{i}\right)_{i \in S}$ with $0<r_{i}<1, i \in S$. We denote by $R(x, y)$ the effective resistance metric induced by $(D, \mathbf{r})$. Under this metric, $V_{*}$ is dense in $K$ Kig01, Section 3.3] and the functions $F_{w}$ become contractions with Lipschitz constant $\operatorname{Lip} F_{w} \leq r_{w}=r_{w_{1}} \cdots r_{w_{m}}$.

Let $\overline{\mathcal{E}}(f, g)$ be the Dirichlet form associated to $(D, \mathbf{r})$, defined on $\mathcal{F} \subset C(K)$. It satisfies, for $m \geq 1$,

$$
\mathcal{E}(f, g)=\sum_{w \in W_{m}} \frac{1}{r_{w}} \mathcal{E}\left(f \circ F_{w}, g \circ F_{w}\right) .
$$

By a theorem of Kumagai [Kum93] (see Kig01, Section 3.4]), for any self-similar measure $\mu$ on $K,(\mathcal{E}, \mathcal{F})$ is a local regular Dirichlet form on $L^{2}(K, \mu)$, and the corresponding non-negative self-adjoint operator $H_{N}$ on $L^{2}(K, \mu)$ has compact resolvent. If we define $\mathcal{F}_{0}=\left\{u \in \mathcal{F}:\left.u\right|_{V_{0}}=0\right\}$, then $\left(\mathcal{E}, \mathcal{F}_{0}\right)$ is a local Dirichlet form on $L^{2}(K, \mu)$, and the corresponding operator $H_{D}$ has also compact resolvent. Moreover, $H_{D}$ is invertible, and $\left(H_{D}\right)^{-1}$ is a compact operator on $L^{2}(K, \mu)$. The operators $-H_{N}$ and $-H_{D}$ are called the Neumann and Dirichlet Laplacians, respectively.

2.3. Maximal function. Let $d$ be the unique real number that satisfies $\sum_{i \in S}\left(r_{i}\right)^{d}=$ 1. $d$ is called the similarity dimension of the harmonic structure $(D, \mathbf{r})$, and it can be proved that $d$ corresponds to the Hausdorff dimension of $K$ with respect to the resistance metric $R$ Kig01, Section 4.2]. If $\mu$ is the self-similar measure on $K$ with weights $\mu_{i}=\left(r_{i}\right)^{d}, i \in S$, it is easy to prove that $\mu\left(B_{\varepsilon}(x)\right) \sim \varepsilon^{d}$ for any $\varepsilon>0$ 
sufficiently small. More precisely, there exist two constants $A_{1}, A_{2}>0$ such that, for any $x \in K$ and sufficiently small $\varepsilon>0$,

$$
A_{1} \varepsilon^{d} \leq \mu\left(B_{\varepsilon}(x)\right) \leq A_{2} \varepsilon^{d} .
$$

See [Sáe02] for details. For $f \in L^{1}(K, \mu)$, we define the operator

$$
M f(x)=\sup _{\varepsilon>0} \frac{1}{\mu\left(B_{\varepsilon}(x)\right)} \int_{B_{\varepsilon}(x)}|f| d \mu,
$$

where $B_{\varepsilon}(x)$ is the ball of radius $\varepsilon$ around $x$ with respect to the effective resistance metric. The following theorem is standard [Ste70, Chapter III].

Theorem 2.1. If $f$ is an integrable function and $M f$ is given by (2.2), then $M f(x)$ is finite a.e. Moreover,

(1) There exists a constant $A>0$ such that, for any $f \in L^{1}(K, \mu)$ and $\alpha>0$,

$$
\mu(\{x \in K: M f(x)>\alpha\}) \leq \frac{A}{\alpha}\|f\|_{L^{1}(K, \mu)} ;
$$

(2) $M$ extends to a bounded operator on $L^{p}(K, \mu)$ for $1<p \leq \infty$.

Remark 2.2. In the case where $K$ is the interval $I=[0,1]$, the functions $F_{1}$ and $F_{2}$ are the contractions $x \mapsto x / 2$ and $x \mapsto x / 2+1 / 2$, and the harmonic structure corresponds to $D=\left(\begin{array}{cc}-1 & 1 \\ 1 & -1\end{array}\right)$ and $r_{1}=r_{2}=1 / 2$, we have that the effective resistance metric $R$ is equal to the standard metric and $\mu$ is the Lebesgue measure in $I$. In such case, (2.2) is the Hardy-Littlewood maximal operator and Theorem 2.1 is the classical maximal function theorem.

Remark 2.3. It is not hard to see that, as in the classical case, Theorem 2.1 is also true for finite Borel measures on $K$. Precisely, if $\nu$ is a finite Borel measure and we define the function $M \nu$ as

$$
M \nu(x)=\sup _{\varepsilon>0} \frac{1}{\mu\left(B_{\varepsilon}(x)\right)} \int_{B_{\varepsilon}(x)}|d \nu|,
$$

then

$$
\mu(\{x \in K: M \nu(x)>\alpha\}) \leq \frac{A}{\alpha}\|\nu\| .
$$

(Cf. Ste70, Chapter III, 4.1].)

2.4. Laplacians. Let $\Delta$ be the Laplacian associated with $(D, \mathbf{r})$ and $\mu$. We denote its domain by $\mathcal{D}$. We also consider the sets

$$
\mathcal{D}_{D}=\left\{u \in \mathcal{D}:\left.u\right|_{V_{0}}=0\right\} \quad \text { and } \quad \mathcal{D}_{N}=\left\{u \in \mathcal{D}: d u=0 \text { on } V_{0}\right\}
$$

where $d f(p)$ is the Neumann derivative of $f$ at the boundary point $p$ Kig01. One can see then that $H_{D}$ is the Friedrich extension of $-\Delta$ on $\mathcal{D}_{D}$, while $H_{N}$ is the Friedrich extension of $-\Delta$ on $\mathcal{D}_{N}$ (see Kig01, Section 3.7]).

For convenience, as in Kig01, will denote by $b$ either $D$ or $N$; so, for instance, $H_{b}$ will denote the operator $H_{D}$ or $H_{N}$, respectively. 
2.5. Dirichlet and Neumann eigenfunctions. Consider the set $E_{b}(\lambda)=\{\phi \in$ $\left.\mathcal{D}_{b}: \Delta \phi=-\lambda \phi\right\}$. If $\operatorname{dim} E_{D}(\lambda) \neq 0, \lambda$ is called a Dirichlet eigenvalue, and the collection of such $\lambda$ is called the Dirichlet spectrum of $\Delta$. If $\operatorname{dim} E_{N}(\lambda) \neq 0$, then $\lambda$ is called a Neumann eigenvalue, and the collection of such $\lambda$ is called the Neumann spectrum of $\Delta$. Both the Dirichlet and Neumann spectra of $\Delta$ are subsets of $[0, \infty)$, and there are $\lambda_{n}^{b}$ and $\phi_{n}^{b} \in E_{b}\left(\lambda_{n}^{b}\right)$ such that

$$
0 \leq \lambda_{1}^{b} \leq \lambda_{2}^{b} \leq \ldots
$$

and $\left\{\phi_{n}^{b}: n \geq 1\right\}$ is a complete orthonormal system for $L^{2}(K, \mu)$. Observe that $\lambda_{1}^{D}>0$. The following theorem is due to Kigami and Lapidus KL93.

Theorem 2.4. If $\rho_{b}(x)=\sum_{\lambda \leq x} \operatorname{dim} E_{b}(\lambda)$,

$$
0<\liminf _{x \rightarrow \infty} \frac{\rho_{b}(x)}{x^{d /(d+1)}} \leq \limsup _{x \rightarrow \infty} \frac{\rho_{b}(x)}{x^{d /(d+1)}}<\infty .
$$

Equation (2.3) is the analogous to Weyl's formula which counts the eigenvalues of the Laplacian on a domain in $\mathbb{R}^{n}$. We will also make use of the following property:

* There exists a constant $C>0$ such that

$$
\|\phi\|_{\infty} \leq C \lambda^{\frac{d}{2(d+1)}}\|\phi\|_{2}, \quad \phi \in E_{b}(\lambda) .
$$

A proof of this can be found in Kig01, Section 4.5].

\section{The heat And Poisson Kernels}

3.1. Heat kernel. For $b=D$ or $N$, the Dirichlet (respectively Neumann) heat kernel is the function $H^{b}: \mathbb{R}_{+} \times K \times K \rightarrow \mathbb{C}$ defined by

$$
H^{b}(t, x, y)=\sum_{n=1}^{\infty} e^{-\lambda_{n}^{b} t} \phi_{n}^{b}(x) \phi_{n}^{b}(y) .
$$

Although the right-hand sum of (3.1) is defined only formally, it is not hard to prove that it converges uniformly on $[T, \infty) \times K \times K$ for any $T>0$, which follows from the results in Section 2.5 (cf. Section [3.2). Moreover, $H^{b}$ is nonnegative, continuous, defines a fundamental solution to the heat equation Kig01, Section 5.1], and

$$
\int_{K} H^{b}(t, x, z) H^{b}(s, z, y) d \mu(z)=H^{b}(t+s, x, y) .
$$

These properties imply that the operator $f \mapsto \mathcal{H}_{t}^{b} f$, where

$$
\mathcal{H}_{t}^{b} f(x)=\int_{K} H^{b}(t, x, y) f(y) d \mu(y),
$$

defined for $t>0$ and integrable functions $f$ on $K$, is a strongly continuous semigroup on $L^{2}(K, \mu)$ whose generator is given by $-H_{b}$. In fact, as $H^{b}$ is continuous and $K$ is compact, we have that $\mathcal{H}_{t}^{b}$ is bounded from $L^{p}(K, \mu)$ to $C(K)$ for any $t>0$ and $1 \leq p \leq \infty$ and, moreover,

$$
\mathcal{H}_{t}^{b}\left(L^{p}(K, \mu)\right) \subset \mathcal{D}_{b}
$$

If $u(t, x)$ is defined on $\mathbb{R}_{+} \times K$ as $u(t, x)=\mathcal{H}_{t}^{b} f(x)$, then

$$
\frac{\partial u(t, x)}{\partial t}=\Delta u(t, x)
$$


so $H^{b}$ is the fundamental solution of the heat equation on $K$. For the details of these facts, see [Kig01, Chapter 5]. The following result is also proved in Kig01, Proposition 5.2.6].

Proposition 3.1. (1) For $f \in C(K),\left\|\mathcal{H}_{t}^{N} f-f\right\|_{L^{\infty}(K, d \mu)} \rightarrow 0$ as $t \rightarrow 0$.

(2) Let $f \in C(K)$, with $\left.f\right|_{V_{0}} \equiv 0$. Then $\left\|\mathcal{H}_{t}^{D} f-f\right\|_{L^{\infty}(K, d \mu)} \rightarrow 0$ as $t \rightarrow 0$.

Thus we observe that the heat kernel acts as an approximation to the identity for continuous functions.

3.2. Poisson kernel. We define the Dirichlet (respectively Neumann) Poisson kernel $P^{b}: \mathbb{R}_{+} \times K \times K \rightarrow \mathbb{C}$ by

$$
P^{b}(t, x, y)=\sum_{n=1}^{\infty} e^{-\sqrt{\lambda_{n}^{b}} t} \phi_{n}^{b}(x) \phi_{n}^{b}(y) .
$$

As above, we first take the series in (3.3) formally. However, the series converges uniformly on $[T, \infty) \times K \times K$ for any $T>0$, which follows from the following two observations.

(1) There exist $c_{1}, c_{2}>0$ such that

$$
c_{1} n^{(d+1) / d} \leq \lambda_{n}^{b} \leq c_{2} n^{(d+1) / d}
$$

(2) For $\alpha, \beta, \gamma, T>0, \sum_{n \geq 1} n^{\alpha} e^{-\gamma n^{\beta} t}$ converges uniformly for $t \in[T, \infty)$.

The first follows from Theorem 2.4 while the second is straightforward from the well-known properties of the exponential function. Now, from these and equation (2.4) it follows that

$$
\left|e^{-\sqrt{\lambda_{n}^{b}} t} \phi_{n}^{b}(x) \phi_{n}^{b}(y)\right| \lesssim e^{-\sqrt{c_{1}} t n^{(d+1) /(2 d)}} n
$$

uniformly in $K \times K$, and thus the series in (3.3) converges uniformly on $[T, \infty) \times$ $K \times K$ for any $T>0$.

The following identity, based on the principle of subordination Ste70, Section III.2], will be useful to study the properties of $P^{b}(t, x, y)$.

Proposition 3.2. For $(t, x, y) \in \mathbb{R}_{+} \times K \times K$,

$$
P^{b}(t, x, y)=\frac{t}{2 \sqrt{\pi}} \int_{0}^{\infty} e^{-t^{2} / 4 s} H^{b}(s, x, y) \frac{d s}{s^{3 / 2}},
$$

where $H^{b}$ is the heat kernel on $K$.

Proof. By the estimates (2.4) and (3.4), we see that

$$
H^{b}(t, x, y) \leq \sum_{n=1}^{\infty} e^{-\lambda_{n}^{b} t}\left(\lambda_{n}^{b}\right)^{d /(d+1)} \leq \sum_{n=1}^{\infty} n e^{-c_{1} n^{(d+1) / d} t},
$$

uniformly on $K \times K$. Let $\alpha=\frac{d}{(d+1)}$. Since, $e^{-c_{1} n^{1 / \alpha} t} \lesssim \frac{1}{\left(n^{1 / \alpha} t\right)^{2 \alpha+1}}$, we see that the series can be estimated by

$$
\sum_{n=1}^{\infty} n e^{-c_{1} n^{1 / \alpha} t} \lesssim \frac{1}{t^{2 \alpha+1}}
$$


Now the integral $\int_{0}^{\infty} e^{-t^{2} / 4 s} \frac{1}{s^{2 \alpha+1}} \frac{d s}{s^{3 / 2}}$ converges for $t>0$, so by the dominated convergence theorem

$$
\begin{aligned}
\int_{0}^{\infty} e^{-t^{2} / 4 s} H^{b}(s, x, y) \frac{d s}{s^{3 / 2}} & =\sum_{n=1}^{\infty} \phi_{n}^{b}(x) \phi_{n}^{b}(y) \int_{0}^{\infty} e^{-t^{2} / 4 s} e^{-\lambda_{n}^{b} s} \frac{d s}{s^{3 / 2}} \\
& =\sum_{n=1}^{\infty} \phi_{n}^{b}(x) \phi_{n}^{b}(y) \frac{2 \sqrt{\pi}}{t} e^{-\sqrt{\lambda_{n}^{b}} t}=\frac{2 \sqrt{\pi}}{t} P^{b}(t, x, y),
\end{aligned}
$$

where we have used the identity

$$
\int_{0}^{\infty} e^{-t^{2} / 4 s} e^{-\beta^{2} s} \frac{d s}{s^{3 / 2}}=\frac{2 \sqrt{\pi}}{t} e^{-\beta t}
$$

for $\beta>0$ [Ste70, Section III.2].

Proposition 3.2 allows us to conclude the following properties of $P^{b}(t, x, y)$, analogously to those of $H^{b}(t, x, y)$.

Corollary 3.3. The Poisson kernel satisfies the following properties.

(1) $P^{b}$ is nonnegative and continuous;

(2) For $(t, x) \in \mathbb{R}_{+} \times K, P^{b}(t, x, \cdot) \in \mathcal{D}_{b}$;

(3) For $(x, y) \in K \times K, P^{b}(\cdot, x, y) \in C^{2}\left(\mathbb{R}_{+}\right)$;

(4) For $(t, x, y) \in \mathbb{R}_{+} \times K \times K$,

$$
\frac{\partial^{2} P^{b}(t, x, y)}{\partial t^{2}}+\left(\Delta P^{b}(t, x, \cdot)\right)(y)=0
$$

and

(5) For $t, s \in \mathbb{R}_{+}, x, y \in K$,

$$
\int_{K} P^{b}(t, x, z) P^{b}(s, z, y) d \mu(z)=P^{b}(t+s, x, y) .
$$

Proof. (1) follows from the nonnegativity of $H^{b}(t, x, y)$ and the fact that the integral in Proposition 3.2 converges absolutely.

To prove (2), observe that $\sum_{n \geq 1} a_{n} \phi_{n}^{b} \in \operatorname{dom}\left(H_{b}\right)$ if and only if

$$
\sum_{n \geq 1}\left|\lambda_{n}^{b} a_{n}\right|^{2}<\infty
$$

which clearly holds for $a_{n}=e^{-\sqrt{\lambda_{n}^{b}} t} \phi_{n}^{b}(x)$ for any given $t>0$ and $x \in K$, because of (2.4). Then $P^{b}(t, x, \cdot) \in \operatorname{dom}\left(H_{b}\right)$ and

$$
H_{b} P^{b}(t, x, \cdot)=\sum_{n=1}^{\infty} \lambda_{n}^{b} e^{-\sqrt{\lambda_{n}^{b}} t} \phi_{n}^{b}(x) \phi_{n}^{b} .
$$

As above, one can verify that this series converges uniformly on $[T, \infty) \times K \times K$, and thus $H_{b} P^{b}(t, x, \cdot) \in C(K)$. Therefore $P^{b}(t, x, \cdot) \in \mathcal{D}_{b}$.

Now fix $x, y \in K$ and set $f_{n}(t)=e^{-\sqrt{\lambda_{n}^{b}} t} \phi_{n}^{b}(x) \phi_{n}^{b}(y)$. Since

$$
\sum_{n=1}^{\infty} f_{n}^{\prime}(t)=-\sum_{n=1}^{\infty} \sqrt{\lambda_{n}^{b}} e^{-\sqrt{\lambda_{n}^{b}} t} \phi_{n}^{b}(x) \phi_{n}^{b}(y)
$$


converges uniformly on $[T, \infty)$ for any $T>0, t \mapsto P^{b}(t, x, y)$ is continuosly differentiable and

$$
\frac{\partial P^{b}(t, x, y)}{\partial t}=\sum_{n=1}^{\infty} f_{n}^{\prime}(t)
$$

Now $f_{n}^{\prime \prime}(t)=\lambda_{n}^{b} e^{-\sqrt{\lambda_{n}^{b}} t} \phi_{n}^{b}(x) \phi_{n}^{b}(y)$, so $\sum_{n=1}^{\infty} f_{n}^{\prime \prime}(t)$ also converges absolutely on $[T, \infty)$ for any $T>0$ and thus $t \mapsto P^{b}(t, x, y)$ is in $C^{2}\left(\mathbb{R}_{+}\right)$, which proves (3).

(4) follows from the fact that

$$
\begin{aligned}
\frac{\partial^{2} P^{b}(t, x, y)}{\partial t^{2}} & =\sum_{n=1}^{\infty} f_{n}^{\prime \prime}(t)=\sum_{n=1}^{\infty} \lambda_{n}^{b} e^{-\sqrt{\lambda_{n}^{b}} t} \phi_{n}^{b}(x) \phi_{n}^{b}(y) \\
& =\left(H_{b} P^{b}(t, x, \cdot)\right)(y)=-\left(\Delta P^{b}(t, x, \cdot)\right)(y) .
\end{aligned}
$$

For (5), it is sufficient to note that (3.6) follows from (3.2), Proposition 3.2 and Fubini's theorem.

3.3. Poisson semigroup. The results from the previous section lead us, analogously to the heat kernel, to define the operators $f \mapsto \mathcal{P}_{t}^{b} f(b=D$ or $N)$ for each $t>0$ as

$$
\mathcal{P}_{t}^{b} f(x)=\int_{K} P^{b}(t, x, y) f(y) d \mu(y),
$$

defined for integrable functions $f$ on $K$. The continuity of $P^{b}$ and the compactness of $K$ imply that $\mathcal{P}_{t}^{b}$ is bounded from $L^{p}(K, \mu)$ to $C(K)$ for any $t>0$ and $1 \leq p \leq \infty$. We also see that $\mathcal{P}_{t}^{b} \circ \mathcal{P}_{s}^{b}=\mathcal{P}_{t+s}^{b}$, which follows from (3.6), so we have that $\left\{\mathcal{P}_{t}^{b}\right\}_{t>0}$ is a semigroup.

We in fact have the following theorem.

Theorem 3.4. Let $f \in L^{p}(K, \mu)$ and define, for $(t, x) \in K$,

$$
u(t, x)=\mathcal{P}_{t}^{b} f(x) .
$$

(1) For each $x \in K, u(\cdot, x) \in C^{\infty}\left(\mathbb{R}_{+}\right)$;

(2) For each $t>0, u(t, \cdot) \in \mathcal{D}_{b}$; and

(3) For each $(t, x) \in \mathbb{R}_{+} \times K$,

$$
\frac{\partial^{2} u(t, x)}{\partial t^{2}}+\Delta u(t, x)=0
$$

Proof. Since, for $f \in L^{p}$,

$$
\int_{K}\left|\phi_{n}^{b}(y) f(y)\right| d \mu(y) \leq C \lambda_{n}^{\frac{d}{2(d+1)}}\|f\|_{L^{p}}
$$

by (2.4), we have that, for every positive integer $m$,

$$
\left\|\sum_{n=1}^{m} e^{-\sqrt{\lambda_{n}^{b}} t} \phi_{n}^{b}(x) \phi_{n}^{b} f\right\|_{L^{1}} \leq C\|f\|_{L^{p}} \sum_{n=1}^{\infty} e^{-\sqrt{\lambda_{n}^{b}} t}\left(\lambda_{n}^{b}\right)^{\frac{d}{d+1}},
$$

so by (3.4) the sum is uniformly bounded in $m$ by $C^{\prime}\|f\|_{L^{p}}$. The dominated convergence theorem implies then that $u(t, x)=\sum_{n=1}^{\infty} a_{n} e^{-\sqrt{\lambda_{n}^{b}} t} \phi_{n}^{b}(x)$, where

$$
a_{n}=\int_{K} \phi_{n}^{b}(y) f(y) d \mu(y) \text {. }
$$


Now fix $x \in K$ and set $\psi_{n}(t)=a_{n} e^{-\sqrt{\lambda_{n}^{b}} t} \phi_{n}^{b}(x)$. For each $k \in \mathbb{N}$,

$$
\psi^{(k)}(t)=a_{n}\left(\lambda_{n}^{b}\right)^{k / 2} e^{-\sqrt{\lambda_{n}^{b}} t} \phi_{n}^{b}(x),
$$

so the series $\sum \psi_{n}^{(k)}(t)$ converges uniformly for $t \in[T, \infty)$, for any $T>0$, by (3.4). It follows that $t \mapsto u(t, x)$ is in $C^{k}\left(\mathbb{R}_{+}\right)$for any $k$, which proves (1).

We now fix $t>0$. For (2), we first need to verify that

$$
\sum_{n=1}^{\infty}\left|a_{n} e^{-\sqrt{\lambda_{n}^{b}} t}\right|^{2}<\infty
$$

But this, again, follows from (3.4) and the fact that $\left|a_{n}\right| \leq C\left(\lambda_{n}^{b}\right)^{\frac{d}{2(d+1)}} \|\left. f\right|_{L^{p}}$. This shows $x \mapsto u(t, x)$ is in $\operatorname{dom}\left(H_{b}\right)$ and

$$
H_{b} u(t, \cdot)=\sum_{n=1}^{\infty} a_{n} \lambda_{n}^{b} e^{-\sqrt{\lambda_{n}^{b}} t} \phi_{n}^{b} .
$$

As above, this series converges uniformly, so we have that $H_{b} u(t, \cdot) \in C(K)$, and we conclude $u(t, \cdot) \in \mathcal{D}_{b}$.

Part (3) follows from the sequence of identities

$$
\frac{\partial^{2} u(t, x)}{\partial t^{2}}=\sum_{n=1}^{\infty} \psi_{n}^{\prime \prime}(t)=\sum_{n=1}^{\infty} a_{n} \lambda_{n}^{b} e^{-\sqrt{\lambda_{n}^{b}} t} \phi_{n}^{b}(x)=H_{b} u(t, x)=-\Delta u(t, x) .
$$

We will call the function $u(t, x)=\mathcal{P}_{t}^{b} f(x)$ the Dirichlet Poisson integral (respectively Neumann Poisson integral) of $f$.

As in the case of the heat semigroup, it is not hard to see that $f \mapsto \mathcal{P}_{t}^{b} f$ is a strongly continuous semigroup on $L^{2}(K, \mu)$, which implies that $u(t, x) \rightarrow f(x)$ as $t \rightarrow 0$ in $L^{2}$. It also acts as an approximation to the identity on continuous functions.

Proposition 3.5. (1) Let $f \in C(K)$ and $u_{N}(t, x)$ its Neumann Poisson integral. Then $\left\|u_{N}(t, \cdot)-f\right\|_{L^{\infty}(K, d \mu)} \rightarrow 0$ as $t \rightarrow 0$.

(2) Let $f \in C(K)$ with $\left.f\right|_{V_{0}} \equiv 0$ and $u_{D}(t, x)$ its Dirichlet Poisson integral. Then $\left\|u_{D}(t, \cdot)-f\right\|_{L^{\infty}(K, d \mu)} \rightarrow 0$ as $t \rightarrow 0$.

Proof. This proposition follows from Propositions 3.1 and 3.2. Indeed, as

$$
\frac{t}{2 \sqrt{\pi}} \int_{0}^{\infty} e^{-t^{2} / 4 s} \frac{d s}{s^{3 / 2}}=\frac{t}{2 \sqrt{\pi}} \int_{0}^{\infty} e^{-\frac{t^{2}}{4} s} s^{1 / 2} \frac{d s}{s}=1,
$$

using Fubini's theorem we obtain

$$
u_{b}(t, x)-f(x)=\frac{t}{2 \sqrt{\pi}} \int_{0}^{\infty} e^{-t^{2} / 4 s}\left(\mathcal{H}_{s}^{b} f(x)-f(x)\right) \frac{d s}{s^{3 / 2}},
$$

where $\mathcal{H}_{s}^{b}$ is the heat semigroup. By Proposition 3.1, for any $f \in C(K)$ and any $\varepsilon>0$ there is $\delta>0$ such that, if $0<s<\delta$,

$$
\left\|\mathcal{H}_{s}^{b} f-f\right\|_{L^{\infty}(K, d \mu)}<\varepsilon
$$


if $b=N$, and for $f \in C(K)$ with $\left.f\right|_{V_{0}} \equiv 0$ if $b=D$. Thus

$$
\begin{aligned}
\left\|u_{b}(t, \cdot)-f\right\|_{L^{\infty}(K, d \mu)} & \leq \frac{t}{2 \sqrt{\pi}} \int_{0}^{\infty} e^{-t^{2} / 4 s}\left\|\mathcal{H}_{s}^{b} f-f\right\|_{L^{\infty}(K, d \mu)} \frac{d s}{s^{3 / 2}} \\
& \leq \frac{t}{2 \sqrt{\pi}} \int_{0}^{\delta} e^{-t^{2} / 4 s} \varepsilon \frac{d s}{s^{3 / 2}}+\frac{t}{2 \sqrt{\pi}} \int_{\delta}^{\infty} e^{-t^{2} / 4 s} M \frac{d s}{s^{3 / 2}}
\end{aligned}
$$

where $M>0$ is such that $\left\|\mathcal{H}_{s}^{b} f-f\right\|_{L^{\infty}(K, d \mu)} \leq M$ uniformly in $s$ ( $f$ is continuous).

Now

and

$$
\frac{t}{2 \sqrt{\pi}} \int_{0}^{\delta} e^{-t^{2} / 4 s} \frac{d s}{s^{3 / 2}} \leq \frac{t}{2 \sqrt{\pi}} \int_{0}^{\infty} e^{-t^{2} / 4 s} \frac{d s}{s^{3 / 2}}=1
$$

$$
\frac{t}{2 \sqrt{\pi}} \int_{\delta}^{\infty} e^{-t^{2} / 4 s} \frac{d s}{s^{3 / 2}} \leq \frac{t}{2 \delta^{1 / 4} \sqrt{\pi}} \int_{0}^{1 / \delta} e^{-t^{2} s / 4} s^{1 / 4} \frac{d s}{s} \leq \frac{\sqrt{t}}{\sqrt{2} \delta^{1 / 4} \sqrt{\pi}} \Gamma(1 / 4),
$$

SO

$$
\left\|u_{b}(t, \cdot)-f\right\|_{L^{\infty}(K, d \mu)} \leq \varepsilon+C \sqrt{t}
$$

for some constant $C>0$. Therefore, as $\varepsilon>0$ is arbitrary, we obtain both cases of the proposition.

We now state and prove the following theorem, which describes the boundary behavior of $u(t, x)$ for $f \in L^{p}$.

Theorem 3.6. Let $f \in L^{p}(K, \mu), 1 \leq p \leq \infty$, and $u(t, x)$ either its Dirichlet or Neumann Poisson integral.

(1) There exists a constant $A>0$ such that, for every $t>0$,

$$
|u(t, x)| \leq A M f(x),
$$

where $M f$ is the maximal function defined in Section 2.3;

(2) $u(t, \cdot) \rightarrow f$ in $L^{p}(K, \mu)$, if $1 \leq p<\infty$;

(3) $\lim _{t \rightarrow 0} u(t, x)=f(x)$ for a.e. $x \in K$.

For the proof of this theorem we use the following Lemma.

Lemma 3.7. There exists a constant $C>0$ such that, for any $x, y \in K$ and $t>0$,

$$
P^{b}(t, x, y) \leq C \min \left\{t^{-\frac{2 d}{d+1}}, \frac{t}{R(x, y)^{\frac{3 d+1}{2}}}\right\} \text {. }
$$

Proof. This lemma follows from the estimate for the heat kernel

$$
H^{b}(t, x, y) \leq A t^{-\frac{d}{d+1}} \exp \left(-c^{\prime}\left(\frac{R(x, y)^{d+1}}{t}\right)^{1 / d}\right)
$$

for some $A, c^{\prime}>0$ [Bar98, Theorem 8.15]. By Proposition 3.2.

$$
P^{b}(t, x, y)=\frac{t}{2 \sqrt{\pi}} \int_{0}^{\infty} e^{-t^{2} / 4 s} H^{b}(s, x, y) \frac{d s}{s^{3 / 2}}
$$

so we have

$$
\begin{aligned}
P^{b}(t, x, y) & \lesssim t \int_{0}^{\infty} e^{-t^{2} / 4 s} s^{-\frac{d}{d+1}} \frac{d s}{s^{3 / 2}}=t \int_{0}^{\infty} e^{-t^{2} / 4 s} s^{-\frac{3 d+1}{2(d+1)}} \frac{d s}{s} \\
& \approx t \cdot t^{-\frac{3 d+1}{d+1}}=t^{-\frac{2 d}{d+1}}
\end{aligned}
$$


and also

$$
P^{b}(t, x, y) \lesssim t \int_{0}^{\infty} \exp \left(-c^{\prime}\left(\frac{R(x, y)^{d+1}}{s}\right)^{1 / d}\right) s^{-\frac{3 d+1}{2(d+1)}} \frac{d s}{s} \lesssim t \cdot R(x, y)^{-\frac{3 d+1}{2}} .
$$

Proof of Theorem 3.6. (1) This part follows by an argument analogous to the well-known Euclidean case: since the Poisson integral $u(t, x)$ of $f$ is given by

we write

$$
u(t, x)=\int_{K} P^{b}(t, x, y) f(y) d \mu(y),
$$

$|u(t, x)| \leq \int_{K} P^{b}(t, x, y)|f(y)| d \mu(y)=\sum_{n=0}^{\infty} \int_{A_{n}(x)} P^{b}(t, x, y)|f(y)| d \mu(y)$,

where $A_{0}(x)=\left\{y \in K: R(x, y) \leq t^{\frac{2}{d+1}}\right\}$, and

$$
A_{n}(x)=\left\{y \in K: 2^{n-1} t^{\frac{2}{d+1}}<R(x, y) \leq 2^{n} t^{\frac{2}{d+1}}\right\}, \quad n \geq 1 .
$$

Now, from Lemma 3.7 and the estimate (2.1),

$$
\begin{aligned}
\int_{A_{0}(x)} P^{b}(t, x, y) \mid & f(y) \mid d \mu(y) \\
& \lesssim t^{-\frac{2 d}{d+1}} \cdot \frac{t^{\frac{2}{d+1}} \cdot d}{\mu\left(B_{t^{2 /(d+1)}}(x)\right)} \int_{B_{t^{2 /(d+1)}(x)}}|f(y)| d \mu(y) \\
& \leq M f(x) .
\end{aligned}
$$

Similarly, for each $n \geq 1$,

$$
\begin{aligned}
\int_{A_{n}(x)} P^{b}(t, x, y) \mid & f(y) \mid d \mu(y) \\
& \lesssim t \int_{A_{n}(x)} \frac{1}{R(x, y)^{\frac{3 d+1}{2}}}|f(y)| d \mu(y) \\
& \leq t \cdot \frac{1}{\left(2^{n-1} t^{\frac{2}{d+1}}\right)^{\frac{3 d+1}{2}}} \int_{B_{2^{n} t^{2 /(d+1)}(x)}|f(y)| d \mu(y)} \\
& \lesssim \frac{t^{-\frac{2 d}{d+1}} 2^{-n \frac{3 d+1}{2}}\left(2^{n} t^{\frac{2}{d+1}}\right)^{d}}{\mu\left(B_{2^{n} t^{2 /(d+1)}}(x)\right)} \int_{B_{2^{n} t^{2 /(d+1)}(x)}}|f(y)| d \mu(y) \\
& \leq 2^{-\frac{d+1}{2} n} M f(x) .
\end{aligned}
$$

Therefore

$$
|u(t, x)| \lesssim \sum_{n=0}^{\infty} 2^{-\frac{d+1}{2} n} M f(x) \lesssim M f(x)
$$

(2) This part follows, as in the classical case, from the fact that the family $\left\{P^{b}(t, x, y)\right\}_{t>0}$ forms an approximation to the identity for continuous functions, and the fact that the maximal function is weakly bounded in $L^{1}$ and bounded in $L^{p}, p>1$.

(3) This is standard SW71. 
Remark 3.8. We observe that we can write the estimates of Lemma 3.7 as

$$
P^{b}(t, x, y) \leq \frac{C^{\prime} t}{\left(t^{2}+R(x, y)^{d+1}\right)^{\frac{3 d+1}{2(d+1)}}},
$$

for some $C^{\prime}>0$ and either $b=N$ or $D$, and thus we have an analogous inequality to the classical Poisson kernel.

Remark 3.9. If $\nu$ is a finite Borel measure on $K$, we can define its Dirichlet and Neumann Poisson integrals $u(t, x)=\mathcal{P}_{t}^{b} \nu(x)$ as

$$
\mathcal{P}_{t}^{b} \nu(x)=\int_{K} P^{b}(t, x, y) d \nu(y) .
$$

The same arguments as in Theorem [3.6(1) imply the estimate $|u(t, x)| \lesssim M \nu(x)$, and hence $u(t, \cdot) \rightarrow \nu$ as $t \rightarrow 0$. In particular, $\|u(t, \cdot)\|_{L^{1}}$ is uniformly bounded.

3.4. Nontangential limits. In this section we discuss nontangential limits of Poisson integrals. We first develop the concept of a cone over a point $x \in K$. From the final remark in the previous section, for $\alpha>0$ we consider the set

$$
\Gamma_{\alpha}(x)=\left\{(t, y) \in \mathbb{R}_{+} \times K: R(x, y)^{d+1}<\alpha t^{2}\right\} .
$$

The set $\Gamma_{\alpha}(x)$ is not properly a cone; however, in the case $d>1$, it contains the intersection of the cone

$$
\left\{(t, y) \in \mathbb{R}_{+} \times K: R(x, y)<\sqrt{\alpha} t\right\}
$$

with set $\left\{(t, y) \in \mathbb{R}_{+} \times K: R(x, y)<1\right\}$, as $R(x, y)^{2}>R(x, y)^{d+1}$ for such points.

We now state the following result.

Theorem 3.10. Let $f \in L^{p}(K, d \mu), 1 \leq p \leq \infty$, and $u(t, x)$ either its Dirichlet or its Neumann Poisson integral. Let $\alpha>0$. Then

(1) There exists $A_{\alpha}>0$ such that, for $x \in K$,

$$
\sup _{(t, y) \in \Gamma_{\alpha}(x)}|u(t, y)| \leq A_{\alpha} M f(x),
$$

where $M f$ is the maximal function of $f$;

(2) For almost every $x \in K$,

$$
\lim _{\substack{(t, y) \rightarrow(0, x) \\(t, y) \in \Gamma_{\alpha}(x)}} u(t, y)=f(x)
$$

Proof. (1) The proof of (1) follows as the one in Theorem 3.6. once we prove that, for $(t, y) \in \Gamma_{\alpha}(x), P^{b}(t, y, z)$ satisfies an estimate as in Lemma 3.7 for $(t, x, z)$, i.e.

$$
P^{b}(t, y, z) \leq C_{\alpha} \min \left\{t^{-\frac{2 d}{d+1}}, \frac{t}{R(x, z)^{\frac{3 d+1}{2}}}\right\},
$$

for some constant $C_{\alpha}>0$. Indeed, if $R(x, y) \leq \frac{1}{2} R(x, z)$,

$$
R(y, z) \geq R(x, z)-R(x, y) \geq \frac{1}{2} R(x, z),
$$

and we have, by Lemma 3.7

$$
P^{b}(t, y, z) \leq \frac{C t}{R(y, z)^{\frac{3 d+1}{2}}} \leq \frac{C^{\prime} t}{R(x, z)^{\frac{3 d+1}{2}}} .
$$


If $R(x, y)>\frac{1}{2} R(x, z)$, since $(t, y) \in \Gamma_{\alpha}(x)$,

$$
\alpha t^{2}>R(x, y)^{d+1}>\frac{1}{2^{d+1}} R(x, z)^{d+1},
$$

and therefore, by Lemma 3.7

$$
\begin{aligned}
P^{b}(t, y, z) & \leq C t^{-\frac{2 d}{d+1}}=\frac{C t}{t^{\frac{3 d+1}{d+1}}} \\
& <\frac{C t}{\left(\frac{1}{\alpha 2^{d+1}}\left(R(x, z)^{d+1}\right)^{\frac{3 d+1}{2(d+1)}}\right.}=\frac{C_{\alpha} t}{R(x, z)^{\frac{3 d+1}{2}}} .
\end{aligned}
$$

The proof now follows as in Theorem 3.6, by decomposing the Poisson integral

$$
u(t, y)=\int_{K} P^{b}(t, y, z) f(z) d \mu(z)
$$

in annuli $A_{n}(x)$, with center $x$, of radius $\sim 2^{n} t^{\frac{2}{d+1}}$ for each $n$.

For the second part of the theorem we need the following lemma.

Lemma 3.11. For any $x \in K \backslash V_{0}$,

$$
\lim _{t \rightarrow 0} \int_{K} P^{D}(t, x, y) d \mu(y)=1 .
$$

Proof. Let $U$ be a neighborhood of $V_{0}$ and $\delta>0$ such that $B_{\delta}(x) \cap U=\emptyset$. Let $f$ be a continuous function on $K$ such that $f \equiv 1$ on $K \backslash U$ and $\left.f\right|_{V_{0}} \equiv 0$. Hence

$$
\int_{K} P^{D}(t, x, y) d \mu(y)=\int_{K} P^{D}(t, x, y) f(y) d \mu(y)+\int_{U} P^{D}(t, x, y)(1-f(y)) d \mu(y) .
$$

By Proposition 3.5.

$$
\int_{K} P^{D}(t, x, y) f(y) d \mu(y) \rightarrow f(x)=1
$$

as $t \rightarrow 0$ and, since $R(x, y)>\delta$ for $y \in U$,

$$
\int_{U} P^{D}(t, x, y)|1-f(y)| d \mu(y) \leq A t \int_{U} \frac{d \mu(y)}{R(x, y)^{\frac{3 d+1}{2}}}<A_{\delta} \mu(U) t .
$$

Therefore

$$
\int_{K} P^{D}(t, x, y) d \mu(y) \rightarrow 1
$$

as $t \rightarrow 0$.

Observe that in the Neumann case one actually has

$$
\int_{K} P^{N}(t, x, y) d \mu(y)=1
$$

for every $t>0$. 
Proof of Theorem 3.10. (2) Let $x \in K \backslash V_{0}$ be in the Lebesgue set of $f$, and let $\varepsilon>0$. Thus, there exists $\delta>0$ such that

$$
\frac{1}{\mu\left(B_{r}(x)\right)} \int_{B_{r}(x)}|f(y)-f(x)| d \mu(y)<\varepsilon
$$

for every $r<\delta$. We thus define the function $g$ on $K$ by

$$
g(y)= \begin{cases}|f(y)-f(x)| & R(x, y)<\delta \\ 0 & R(x, y) \geq \delta .\end{cases}
$$

Hence (3.9) implies $M g(x)<\varepsilon$. We now have

$$
\begin{aligned}
& |u(t, y)-f(x)| \leq \\
& \quad \int_{K} P^{b}(t, y, z)|f(z)-f(x)| d \mu(z)+\left|\int_{K} P^{b}(t, y, z) d \mu(z)-1\right| \cdot|f(x)| .
\end{aligned}
$$

By Lemma 3.11, the second term in the right hand side is either zero in the Neumann case $b=N$, or goes to 0 as $t \rightarrow 0$ in the Dirichlet case $b=D$.

We split the first term as the sum

$$
\int_{B_{\delta}(x)} P^{b}(t, y, z)|f(z)-f(x)| d \mu(z)+\int_{K \backslash B_{\delta}(x)} P^{b}(t, y, z)|f(z)-f(x)| d \mu(z) .
$$

Now, by part (1) of the theorem, if $(t, y) \in \Gamma_{\alpha}(x)$,

$$
\begin{aligned}
\int_{B_{\delta}(x)} P^{b}(t, y, z)|f(z)-f(x)| d \mu(z) & =\int_{K} P^{b}(t, y, z) g(z) d \mu(z) \\
& \leq A_{\alpha} M g(x)<A_{\alpha} \varepsilon .
\end{aligned}
$$

Also, by (3.8), if $(t, y) \in \Gamma_{\alpha}(x)$,

$$
\begin{aligned}
\int_{K \backslash B_{\delta}(x)} P^{b}(t, y, z)|f(z)-f(x)| d \mu(z) & \leq C_{\alpha} t \int_{K \backslash B_{\delta}(x)} \frac{|f(z)-f(x)|}{R(x, z)^{\frac{3 d+1}{2}}} d \mu(z) \\
& \leq C_{\alpha, \delta} t \int_{K}|f(z)-f(x)| d \mu(z) \\
& \leq C_{\alpha, \delta}\left(\|f\|_{L^{p}}+|f(x)|\right) t,
\end{aligned}
$$

and thus goes to 0 as $t \rightarrow 0$. Hence we have

$$
\limsup _{\substack{(t, y) \rightarrow(0, x) \\(t, y) \in \Gamma_{\alpha}(x)}}|u(t, y)-f(x)| \leq A_{\alpha} \varepsilon
$$

and, as $\varepsilon>0$ is arbitrary,

$$
\lim _{\substack{(t, y) \rightarrow(0, x) \\(t, y) \in \Gamma_{\alpha}(x)}} u(t, y)=f(x)
$$

Since $V_{0}$ is finite and the Lebesgue set of $f$ contains almost every point of $K$ (if $f \in L^{p}(K, d \mu)$, then $f$ is integrable, as we have noted above), therefore we obtain the theorem. 


\section{FAtou-type theorems}

4.1. A maximum principle. We say that a continuous function $u$ on $\mathbb{R}_{+} \times K$ is a harmonic function if

(1) $u(\cdot, x) \in C^{2}\left(\mathbb{R}_{+}\right)$for each $x \in K$;

(2) $u(t, \cdot) \in \mathcal{D}$ for each $t>0$; and

(3) For each $(t, x) \in \mathbb{R}_{+} \times K \backslash V_{0}$,

$$
\frac{\partial^{2} u(t, x)}{\partial t^{2}}+\Delta u(t, x)=0
$$

For example, if $u(t, x)$ is the Poisson integral of $f \in L^{p}(K, d \mu)$, then $u$ is a harmonic function, by Theorem 3.4 .

We state and prove the following result, analogous to the parabolic maximum principle for solutions of the heat equation Kig01, Section 5.2].

Theorem 4.1. Let $u$ be a harmonic function on $\mathbb{R}_{+} \times K$. Then $u$ cannot take $a$ maximum in $\mathbb{R}_{+} \times\left(K \backslash V_{0}\right)$.

As in the case of the parabolic maximum principle, the proof of Theorem 4.1 makes use of the following lemma, whose proof can be found in Kig01, Lemma 5.2.4].

Lemma 4.2. Let $u \in \mathcal{D}$. If $u(x)=\max \{u(y): y \in K\}$ for some $x \in K \backslash V_{0}$, then $\Delta u(x) \leq 0$.

Proof of Theorem 4.1. For $n \in \mathbb{Z}_{+}$, set $u_{n}=u+t^{2} / n$. Suppose $u_{n}$ takes its maximum at an interior point $\left(t_{0}, x_{0}\right) \in \mathbb{R}_{+} \times\left(K \backslash V_{0}\right)$. Then

$$
\frac{\partial^{2} u_{n}}{\partial t^{2}}\left(t_{0}, x_{0}\right) \leq 0 \text {. }
$$

But

so

$$
\frac{\partial^{2} u_{n}}{\partial t^{2}}=\frac{\partial^{2} u}{\partial t^{2}}+\frac{2}{n}=-\Delta u+\frac{2}{n},
$$

$$
\Delta u\left(t_{0}, x_{0}\right)=-\frac{\partial^{2} u_{n}}{\partial t^{2}}\left(t_{0}, x_{0}\right)+\frac{2}{n}>0,
$$

contradicting Lemma 4.2 because the function

$$
x \mapsto u\left(t_{0}, x\right)=u_{n}\left(t_{0}, x\right)-\frac{t_{0}^{2}}{n}
$$

takes its maximum at $x_{0} \in K \backslash V_{0}$.

From Theorem 4.1, if $u$ is a bounded continuous function on $[0, \infty) \times K$ which is harmonic on $\mathbb{R}_{+} \times K$, then, if $u$ takes its maximum in $\left(t_{0}, x_{0}\right)$, then $t_{0}=0$ or $x_{0} \in V_{0}$.

Moreover, by taking $-u$, we can similarly conclude that its minimum, if taken by $u$ at $\left(t_{0}, x_{0}\right)$, must satisfy $t_{0}=0$ or $x_{0} \in V_{0}$. We have the following corollary.

Corollary 4.3. Let $u$ be continuous in $[0, \infty) \times K$, harmonic on $\mathbb{R}_{+} \times K$, and $0 \leq a<b$. If $u(a, x) \geq 0$ and $u(b, x) \geq 0$ for every $x \in K$, and $u(t, p) \geq 0$ for every $p \in V_{0}$ and $a \leq t \leq b$, then $u(t, x) \geq 0$ for every $(t, x) \in[a, b] \times K$.

We can clearly extend this corollary to any open set in $\mathbb{R}_{+} \times K$. 
Corollary 4.4. Let $u$ be continuous in $[0, \infty) \times K$, harmonic on $\mathbb{R}_{+} \times K$ and $\Omega \subset \mathbb{R}_{+} \times K$ an open set. If $u(t, x) \geq 0$ for $(t, x) \in \partial \Omega$, then $u(t, x) \geq 0$ for every $(t, x) \in \Omega$.

4.2. Harmonic functions and boundary limits. We have observed that, if $u(t, x)$ is either the Dirichlet or Neumann Poisson integral of $f \in L^{p}(K, d \mu)$, then $u$ is a harmonic function on $\mathbb{R}_{+} \times K$. Moreover, Theorem 3.6 implies that

$$
\sup _{t>0}\|u(t, \cdot)\|_{L^{p}(K, d \mu)}<\infty
$$

if $1 \leq p \leq \infty$. We now prove the converse for uniformly bounded Dirichlet harmonic functions, a result analogous to the classical Fatou's theorem ABR01.

One can easily observe that if $u(t, x)$ is the Dirichlet Poisson integral of a function on $K$, then $u(t, p)=0$ for every $p \in V_{0}$ and $t>0$, as the Dirichlet Poisson kernel satisfies $P^{D}(t, p, y)=0$ for $p \in V_{0}$.

We say that a continuous function $u$ on $\mathbb{R}_{+} \times K$ is a Dirichlet harmonic function if $u$ is harmonic and $u(t, p)=0$ for every $t>0$ and $p \in V_{0}$.

Theorem 4.5. Let $u$ be a Dirichlet harmonic function on $\mathbb{R}_{+} \times K$ such that

$$
\sup _{t>0}\|u(t, \cdot)\|_{L^{\infty}(K, d \mu)}<\infty .
$$

Then $u$ is the Dirichlet Poisson integral of a function $f \in L^{\infty}(K, d \mu)$.

Proof. Let $u$ be a Dirichlet bounded harmonic function on $\mathbb{R}_{+} \times K$, and $M>0$ such that $|u(t, x)| \leq M$ for every $(t, x) \in \mathbb{R}_{+} \times K$.

For $x \in K$ and $n \in \mathbb{Z}_{+}$, set $f_{n}(x)=u(1 / n, x)$, and let $u_{n}(t, x)$ be the Dirichlet Poisson integral of $f_{n}$. We define then, for $(t, x) \in \mathbb{R}_{+} \times K$,

$$
U_{n}(t, x)=u\left(t+\frac{1}{n}, x\right)-u_{n}(t, x)
$$

We claim that $U_{n}(t, x) \equiv 0$. First note that $U_{n}$ is bounded, since $|u| \leq M$ and, by Theorem $3.6(1)$,

$$
\left|u_{n}(t, x)\right| \leq A M f_{n}(x) \leq A\left\|M f_{n}\right\|_{L^{\infty}(K)} \leq A|| f_{n} \|_{L^{\infty}(K)} \leq A M .
$$

Thus $\left|U_{n}(t, x)\right| \leq(A+1) M=A^{\prime} M$. Moreover, since $f_{n}$ is continuous, $u_{n}(t, x)$ can be extended to $t=0$ with $u_{n}(0, x)=f_{n}(x)$, and hence

$$
U_{n}(0, x)=u\left(\frac{1}{n}, x\right)-f_{n}(x)=0
$$

for every $x \in K$. Fix $\left(t_{0}, x_{0}\right) \in \mathbb{R}_{+} \times K$ and $\varepsilon>0$ such that $\frac{1}{\varepsilon}>t_{0}$. Define, for $(t, x) \in[0, \infty) \times K$,

$$
U(t, x)=U_{n}(t, x)+A^{\prime} M \varepsilon t .
$$

Then $U$ is continuous in $[0, \infty) \times K$ and harmonic on $\mathbb{R}_{+} \times K$. Moreover, for $t=0$, $U(0, x)=0$ for every $x \in K$ and, for $t=\frac{1}{\varepsilon}$,

$$
U\left(\frac{1}{\varepsilon}, x\right)=U_{n}\left(\frac{1}{\varepsilon}, x\right)+A^{\prime} M \geq 0,
$$

since $\left|U_{n}(t, x)\right| \leq A^{\prime} M$. Finally, as $U_{n}(t, p)=0$ for $p \in V_{0}$, we have

$$
U(t, p)=A^{\prime} M t \varepsilon \geq 0
$$


for $p \in V_{0}$. Therefore, by Corollary 4.3. $U(t, x) \geq 0$, and hence

$$
U_{n}\left(t_{0}, x_{0}\right) \geq-A^{\prime} M \varepsilon t_{0} .
$$

Since $\varepsilon$ is arbitrary, $U_{n}\left(t_{0}, x_{0}\right) \geq 0$.

Similarly, taking $-U_{n}(t, x)$, we can conclude that $U_{n}\left(t_{0}, x_{0}\right) \leq 0$, and therefore $U_{n}\left(t_{0}, x_{0}\right)=0$ for any $\left(t_{0}, x_{0}\right) \in[0, \infty) \times K$.

This shows that, for any $n, u(t+1 / n, x)$ is the Dirichlet Poisson integral of $f_{n}$, i.e.

$$
u\left(t+\frac{1}{n}, x\right)=\int_{K} P^{D}(t, x, y) f_{n}(y) d \mu(y) .
$$

Now, for every $n,\left\|f_{n}\right\|_{L^{\infty}} \leq M$, so by the weak-* compactness of the ball in $L^{\infty}(K, d \mu)$, there is a subsequence $f_{n_{k}} \rightarrow f$ weakly in $L^{\infty}(K, d \mu)$, and hence

$$
\int_{K} \psi(y) f_{n_{k}}(y) d \mu(y) \rightarrow \int_{K} \psi(y) f(y) d \mu(y)
$$

for any $\psi \in L^{1}(K, d \mu)$. Taking, for each $t>0$ and $x \in K, \psi(y)=P^{D}(t, x, y)$, we obtain, by the continuity of $u(t, x)$, that

$$
u(t, x)=\int_{K} P^{D}(t, x, y) f(y) d \mu(y) .
$$

Therefore $u(t, x)$ is the Dirichlet Poisson integral of the $L^{\infty}$ function $f$.

So we finally have, from Theorems 3.10 and 4.5 the following corollary.

Corollary 4.6. Then a bounded Dirichlet harmonic function on $\mathbb{R}_{+} \times K$ has nontangential limit at $x$ as $t \rightarrow 0$ for almost every $x \in K$.

As in the classical setting [Ste70, Chapter VII], Theorem 4.5 can be extended to the case $1 \leq p<\infty$. Together with Theorem 3.6 and Remark 3.9] we obtain the following Corollary.

Corollary 4.7. Suppose $u$ is a Dirichlet harmonic function on $\mathbb{R}_{+} \times K$. If, $1<$ $p \leq \infty$, then $u$ is the Poisson integral of some $f \in L^{p}$ if and only if

$$
\sup _{t>0}\|u(t, \cdot)\|_{L^{p}}<\infty .
$$

Moreover, $u$ is the Poisson integral of some finite Borel measure on $K$ if and only if

$$
\sup _{t>0}\|u(t, \cdot)\|_{L^{1}}<\infty .
$$

Proof. If $u$ is the Dirichlet Poisson integral of either some $f \in L^{p}$ or a finite Borel measure on $K$, the estimates follow from Theorem 3.6 and Remark 3.9.

Now, suppose $p<\infty$ and $\sup _{t>0}\|u(t, \cdot)\|_{L^{p}}<\infty$. Since $\left\{\mathcal{P}_{t}^{D}\right\}_{t>0}$ is a semigroup, for any $t>t_{0}>0$,

$$
|u(t, x)| \leq \int_{K} P^{D}\left(t-t_{0}, x, y\right)\left|u\left(t_{0}, y\right)\right| d \mu(y) \lesssim\left(\int_{K}\left|P^{D}\left(t-t_{0}, x, y\right)\right|^{q} d \mu(y)\right)^{1 / q},
$$

where $q$ is the conjugate exponent to $p$, independently of $t_{0}$ because $\|u(t, \cdot)\|_{L^{p}}$ is uniformly bounded in $t>0$. From Lemma 3.7 and the same decomposition as in the proof of Theorem 3.6, one proves

$$
\left(\int_{K}\left|P^{D}\left(t-t_{0}, x, y\right)\right|^{q} d \mu(y)\right)^{1 / q} \lesssim\left(t-t_{0}\right)^{-\frac{2 d}{d+1} \cdot \frac{1}{p}}
$$


uniformly in $t, t_{0}$ and $x$, so we have $u(t, x) \lesssim t^{-\frac{2 d}{d+1} \cdot \frac{1}{p}}$ uniformly in $x$. By Theorem 4.5. $u(t+1 / k, x)=\mathcal{P}^{D} f_{k}(x)$, where $f_{k}(x)=u(1 / k, x)$. Since, by assumption, the $L^{p}$ norms of $f_{k}$ are uniformly bounded, the Corollary follows from a similar weak-* argument as in the proof of Theorem 4.5.

\section{A local Fatou theorem}

In this section we prove a local Fatou theorem, analogous to the classical nontangential convergence at the boundary of nontangentially bounded harmonic functions ([Ste70, Thm. VII.3], ABR01, Thm. 7.30]).

As we'll need estimates from below for the Neumann Poisson kernel, we can only prove these for the so called nested fractals Lin90, which we define below.

5.1. Nested fractals. We begin by defining the concept of affine nested fractals. We now assume that $\left(K, S,\left\{F_{i}\right\}_{i \in S}\right)$ is a connected post-critically finite self-similar set, $K \subset \mathbb{R}^{n}$ and each $F_{i}$ is the restriction to $\mathbb{R}^{n}$ of a similitude $F_{i}: \mathbb{R}^{n} \rightarrow \mathbb{R}^{n}$, that is, a map of the form $x \mapsto c U x+a$, where $0<c<1, a \in \mathbb{R}^{n}$ and $U \in O(n)$.

We say that a homeomorfism $f: K \rightarrow K$ is a symmetry of $\left(K, S,\left\{F_{i}\right\}_{i \in S}\right)$ if, for every $m \geq 0$, there exists a map $f_{m}: W_{m} \rightarrow W_{m}$ such that

$$
f\left(F_{w}\left(V_{0}\right)\right)=F_{f_{m}(w)}\left(V_{0}\right), \quad \text { for every } w \in W_{m} .
$$

That is, a symmetry preserves the self-similar structure of $K$.

For $x, y \in \mathbb{R}^{n}$, let $H_{x y}$ be the bisecting hyperplane of the segment from $x$ to $y$, and $\psi_{x y}: \mathbb{R}^{n} \rightarrow \mathbb{R}^{n}$ be the reflection with respect to $H_{x y}$.

Definition 5.1.1. We say that $\left(K, S,\left\{F_{i}\right\}_{i \in S}\right)$ is an affine nested fractal if $\left.\psi_{x y}\right|_{K}$ is a symmetry for any $x, y \in V_{0}$.

In other words, reflecting $K$ by each pair of points in its boundary $V_{0}$ preserves its self-similar structure. Examples of affine nested fractals are the Sierpinski gasket, the Vicsek set or the pentakun, among others (see [Kig01, Section 3.8] for more examples and a through discussion of affine nested fractals).

It can be proven Kig01, Section 3.8] that if $\left(K, S,\left\{F_{i}\right\}_{i \in S}\right)$ is an affine nested fractal, then there exist a harmonic structure with all $r_{i}$ equal to each other, say $r_{i}=r, i \in S$. We assume this harmonic structure is regular, i. e. $r<1$.

The main result of interest to us is the following theorem [FHK94.

Theorem 5.1. If $H^{N}$ is the Neumann heat kernel on the affine nested fractal $K$, there exist positive constants $c_{1}, c_{2}, c_{3}, c_{4}$ such that

$$
\begin{aligned}
& c_{1} t^{-d /(d+1)} \exp \left(-c_{2}\left(\frac{R(x, y)^{d+1}}{t}\right)^{1 /\left(d_{w}-1\right)}\right) \leq H^{N}(t, x, y) \\
& \leq c_{3} t^{-d /(d+1)} \exp \left(-c_{4}\left(\frac{R(x, y)^{d+1}}{t}\right)^{1 /\left(d_{w}-1\right)}\right),
\end{aligned}
$$

where $d_{w}$ is the walk dimension with respect to shortest path metric.

A discussion on the shortest path metric and walk dimension can be found in Bar98. From Theorem 5.1 we obtain the following integral estimate for the Neumann Poisson kernel. 
Corollary 5.2. Let $P^{N}$ be the Neumann Poisson kernel on the affine nested fractal $K$, and $\alpha>0$. Then there exists $c_{\alpha}>0$ such that

$$
\int_{B} P^{N}(t, x, y) d \mu(y) \geq c_{\alpha}
$$

where $B=B_{\left(\alpha t^{2}\right)^{1 /(d+1)}}(x)$ is the ball of radius $\left(\alpha t^{2}\right)^{\frac{1}{d+1}}$ with center in $x$.

Proof. From Theorem [5.1 we have

$$
\begin{aligned}
P^{N}(t, x, y) & =\frac{t}{2 \sqrt{\pi}} \int_{0}^{\infty} e^{-t^{2} / 4 s} H^{N}(s, x, y) \frac{d s}{s^{3 / 2}} \\
& \gtrsim t \int_{0}^{\infty} e^{-t^{2} / 4 s} s^{-\frac{3 d+1}{2(d+1)}} e^{-c\left(\frac{R(x, y) d+1}{s}\right)^{1 /\left(d_{w}-1\right)}} \frac{d s}{s} .
\end{aligned}
$$

Thus

$$
\begin{aligned}
\int_{B} P^{N}(t, x, y) d \mu(y) & \gtrsim \int_{B} t \int_{0}^{\infty} e^{-t^{2} / 4 s} s^{-\frac{3 d+1}{2(d+1)}} e^{-c\left(\frac{R(x, y) d+1}{s}\right)^{1 /\left(d_{w}-1\right)}} \frac{d s}{s} d \mu(y) \\
& \geq t \int_{B} \int_{0}^{\infty} e^{-t^{2} / 4 s} s^{-\frac{3 d+1}{2(d+1)}} e^{-c\left(\frac{\alpha t^{2}}{s}\right)^{1 /\left(d_{w}-1\right)}} \frac{d s}{s} d \mu(y),
\end{aligned}
$$

since $R(x, y)^{d+1}<\alpha t^{2}$ for $y \in B$.

Now, after the change of variables $s \mapsto \alpha t^{2} s$, we finally obtain

$$
\begin{aligned}
\int_{B} P^{N}(t, x, y) & d \mu(y) \\
& \gtrsim t \cdot \alpha^{-\frac{3 d+1}{2(d+1)}} t^{-\frac{3 d+1}{d+1}} \int_{B} \int_{0}^{\infty} e^{-1 / 4 \alpha s} s^{-\frac{3 d+1}{2(d+1)}} e^{-c / s^{1 /(d w-1)}} \frac{d s}{s} d \mu(y) \\
& =\alpha^{-\frac{3 d+1}{2(d+1)}} t^{-\frac{2 d}{d+1}} \int_{0}^{\infty} e^{-1 / 4 \alpha s} s^{-\frac{3 d+1}{2(d+1)}} e^{-c / s^{1 /(d w-1)}} \frac{d s}{s} \int_{B} d \mu(y) \\
& \geq c_{\alpha}>0
\end{aligned}
$$

because the integral in $s$ converges (and its positive) and

$$
\int_{B} d \mu(y)=\mu\left(B_{\left(\alpha t^{2}\right)^{1 /(d+1)}}(x)\right) \sim\left(\alpha t^{2}\right)^{\frac{d}{d+1}},
$$

by (2.1).

Note that, in fact, $c_{\alpha}=c \alpha^{1 / 2}$. Corollary $[5.2$ allows us to construct a harmonic function, with nontangential limit zero, bounded away from zero at the boundary of a union of truncated cones. We define a truncated cone on $\mathbb{R}_{+} \times K$, for $h, \alpha>0$ and $x \in K$, as the set

$$
\Gamma_{\alpha}^{h}(x)=\left\{(t, y) \in \mathbb{R}_{+} \times K: R(x, y)^{d+1}<\alpha t^{2}, 0<y<h\right\} .
$$

Lemma 5.3. Let $E \subset K$ be a measurable set, $\alpha>0$, and $\Omega=\bigcup_{x \in E} \Gamma_{\alpha}^{1}(x)$. Then there exists a positive harmonic function $v$ on $\mathbb{R}_{+} \times K$ such that

(1) $v \geq 1$ on $(\partial \Omega) \cap\left(\mathbb{R}_{+} \times K\right)$; and

(2) $v$ has nontangential limit 0 at almost every point of $E$.

Proof. Let $P^{N}$ be the Neumann Poisson kernel on $K$ and define the function $w$ on $\mathbb{R}_{+} \times K$ by

$$
w(t, x)=\int_{K} P^{N}(t, x, y) \chi_{K \backslash E}(y) d \mu(y)+t,
$$


where $\chi_{K \backslash E}$ is the characteristic function of $K \backslash E$.

We see that $w \geq 0$ and, by Theorem 3.10, $w$ has nontangential limit 0 at almost every point of $E$, so we need to verify that there exists $\delta>0$ such that $w \geq \delta$ on $(\partial \Omega) \cap\left(\mathbb{R}_{+} \times K\right)$. Clearly, $w(1, x) \geq 1$ for every $x \in K$.

Now, we observe that $(t, y) \in \Gamma_{\alpha}(x)$ if and only if $R(x, y)<\left(\alpha t^{2}\right)^{\frac{1}{d+1}}$, that is $x \in B=B_{\left(\alpha t^{2}\right)^{1 /(d+1)}}(y)$, the ball of radius $\left(\alpha t^{2}\right)^{\frac{1}{d+1}}$ with center $y$. Hence, if $(t, y) \in \partial \Omega, x \notin B$ for every $x \in E$, and thus $B \subset K \backslash E$.

We thus obtain, by Corollary 5.2 .

$$
\begin{aligned}
\int_{K} P^{N}(t, x, y) \chi_{K \backslash E}(y) d \mu(y) & =\int_{K \backslash E} P^{N}(t, x, y) d \mu(y) \geq \int_{B} P^{N}(t, x, y) d \mu(y) \\
& \geq c_{\alpha}>0 .
\end{aligned}
$$

Therefore, if we choose $M=\max \left\{1 / c_{\alpha}, 1\right\}$, the function $v=M w$ satisfies the properties required.

\subsection{Nontangentially bounded functions.}

Definition 5.2.1. Let $u$ be a function on $\mathbb{R}_{+} \times K$. We say that $u$ is nontangentially bounded at $x \in K$ if $u$ is bounded on some $\Gamma_{\alpha}^{h}(x)$.

Note that this definition involves only one truncated cone for each $x$, while the definition of nontangential limit involves all cones over $x$, regardless of their apperture.

We also observe that, in the case where $u$ is continuous on $\mathbb{R}_{+} \times K$, then $u$ is nontangentially bounded at $x$ if and only if is bounded in some $\Gamma_{\alpha}^{1}(x)$.

We now prove the following theorem, analog to the classical local Fatou Theorem ABR01. Remember that we assume that $K$ is an affine nested fractal.

Theorem 5.4. Let $u$ be harmonic on $\mathbb{R}_{+} \times K$ and nontangentially bounded at each point in the set $E \subset K$. Then u has a nontangential limit at almost every point of E.

Proof. Analogously to the classical case, we prove this theorem in a sequence of steps. For each positive integer $k$, define the set

$$
E_{k}=\left\{x \in K:|u(t, y)| \leq k \text { for }(t, y) \in \Gamma_{1 / k}^{1}(x)\right\} .
$$

As $u$ is continuous on $\mathbb{R}_{+} \times K$, each $E_{k}$ is closed and $E=\bigcup_{k} E_{k}$.

Step 1. $u$ is bounded on $\Gamma_{\alpha}^{1}(x)$ for every $\alpha>0$ and for almost every $x \in E_{k}$.

Observe that, if $x \in E_{k}$ is in the Lebesgue set of $\chi_{E_{k}}$, then

$$
\lim _{r \rightarrow 0} \frac{\mu\left(B_{r}(x) \cap E_{k}\right)}{\mu\left(B_{r}(x)\right)}=1 .
$$

Recall also that there exist constants $A_{1}, A_{2}>0$ such that, for sufficiently small $r>0$ (say, $r<\bar{r}$ ),

$$
A_{1} r^{d} \leq \mu\left(B_{r}(x)\right) \leq A_{2} r^{d} .
$$

Let $x \in E_{k}$ be in the Lebesgue set of $\chi_{E_{k}}$. As $u$ is continuous on $\mathbb{R}_{+} \times K$, it is sufficient to prove that, for each $\alpha>0$, there exists $h>0$ such that $\Gamma_{\alpha}^{h}(x) \subset$ $\bigcup_{z \in E_{k}} \Gamma_{1 / k}^{1}(z)$. It is of course sufficient to consider the case $\alpha>1 / k$. 
Let $\delta>0$ such that $\delta<\min \left\{\bar{r}, k^{-1 /(d+1)}\right\}$ and, for $0<r \leq \delta$,

$$
\frac{\mu\left(B_{r}(x) \cap E_{k}\right)}{\mu\left(B_{r}(x)\right)}>1-\frac{A_{1}}{A_{2}}\left(\frac{k^{-1 /(d+1)}}{\alpha^{1 /(d+1)}+k^{-1 /(d+1)}}\right)^{d} .
$$

Set $h=\left(\frac{\delta}{2 \alpha^{1 /(d+1)}}\right)^{(d+1) / 2} \cdot$ Thus $h<1$ and we shall prove $\Gamma_{\alpha}^{h}(x) \subset \Omega$.

We first observe that, if $(t, y) \in \Gamma_{\alpha}^{h}(x)$, then

$$
B_{\left(t^{2} / k\right)^{1 /(d+1)}}(y) \cap E \neq \emptyset \text {. }
$$

Indeed, if $(t, y) \in \Gamma_{\alpha}^{h}(x)$, then $R(x, y)<\left(\alpha t^{2}\right)^{1 /(d+1)}$ and $0<t<h$. Now, for $z \in B_{\left(t^{2} / k\right)^{1 /(d+1)}}(y), R(y, z)<\left(t^{2} / k\right)^{1 /(d+1)}$ and hence

$$
R(x, z)<\left(\alpha^{1 /(d+1)}+k^{-1 /(d+1)}\right) t^{2 /(d+1)},
$$

so $z \in B_{r}(y)$, where $r=\left(\alpha^{1 /(d+1)}+k^{-1 /(d+1)}\right) t^{2 /(d+1)}$. Therefore

$$
B_{\left(t^{2} / k\right)^{1 /(d+1)}}(y) \subset B_{r}(y) .
$$

If $B_{\left(t^{2} / k\right)^{1 /(d+1)}}(y) \cap E_{k}=\emptyset$, then $B_{r}(x) \cap E_{k} \subset B_{r}(x) \backslash B_{\left(t^{2} / k\right)^{1 /(d+1)}}(y)$ and thus

$$
\begin{aligned}
\frac{\mu\left(B_{r}(x) \cap E_{k}\right)}{\mu\left(B_{r}(x)\right)} & \leq \frac{\mu\left(B_{r}(x) \backslash B_{\left(t^{2} / k\right)^{1 /(d+1)}}(y)\right)}{\mu\left(B_{r}(x)\right)}=1-\frac{\mu\left(B_{\left(t^{2} / k\right)^{1 /(d+1)}}(y)\right)}{\mu\left(B_{r}(x)\right)} \\
& \leq 1-\frac{A_{1}\left(\left(t^{2} / k\right)^{1 /(d+1)}\right)^{d}}{A_{2} r^{d}}=1-\frac{A_{1}}{A_{2}}\left(\frac{k^{-1 /(d+1)}}{\alpha^{1 /(d+1)}+k^{-1 /(d+1)}}\right)^{d},
\end{aligned}
$$

which contradicts (5.2) since

$$
r=\left(\alpha^{1 /(d+1)}+k^{-1 /(d+1)}\right) t^{2 /(d+1)}<\left(\alpha^{1 /(d+1)}+k^{-1 /(d+1)}\right) h^{2 /(d+1)}<\delta,
$$

by the choice of $h$.

Hence there exists $x_{0} \in B_{\left(t^{2} / k\right)^{1 /(d+1)}}(y) \cap E_{k}$, which implies $(t, y) \in \Gamma_{1 / k}^{1}\left(x_{0}\right)$, as desired. This finishes the proof of Step 1.

Therefore, there is a subset $F \subset E$ such that $\mu(E \backslash F)=0$ and $u$ is bounded in every cone $\Gamma_{\alpha}^{1}(x)$ for $\alpha>0$ and $x \in F$.

In particular, for a fixed $\alpha>0, u$ is bounded in every $\Gamma_{\alpha}^{1}(x)$ for every $x \in F$. We can thus write $F=\bigcup_{k} F_{k}$, with

$$
F_{k}=\left\{x \in F:|u(t, y)| \leq k \text { for }(t, y) \in \Gamma_{\alpha}^{1}(x)\right\} .
$$

Step 2. At almost every $x \in F_{k}$, the limit

$$
\lim _{\substack{(t, y) \rightarrow(0, x) \\(t, y) \in \Gamma_{\alpha}(x)}} u(t, y)
$$

exists. That is, $u$ has a limit at the boundary point $x$ within the cone $\Gamma_{\alpha}(x)$.

Let $\Omega=\bigcup_{x \in F_{k}} \Gamma_{\alpha}^{1}(x)$. As $u$ is continuous on $\mathbb{R}_{+} \times K$, we may assume, say, $|u| \leq 1$ on the set $\Omega^{\prime}=\bigcup_{x \in F_{k}} \Gamma_{\alpha}^{2}(x)$. Without loss of generality, we can also assume $u$ is real valued.

Now, for each $n \geq 1$, let

$$
G_{n}=\left\{y \in K: \text { there exists } x \in F_{k} \text { such that } R(x, y)^{d+1}<\alpha / n^{2}\right\} .
$$


Each $G_{n}$ is open and $G_{n} \subset F_{k}$. Now define the functions $f_{n}$ on $K$ by

$$
f_{n}(x)=\chi_{G_{n}}(x) u(1 / n, x) .
$$

As $(1 / n, x) \in \Omega$ if and only if $x \in G_{n}$, we have $\left|f_{n}\right| \leq 1$, and thus, passing to a subsequence, $\left(f_{n}\right)$ weakly-* converges to some $f \in L^{\infty}(K, d \mu)$, in particular

$$
\mathcal{P}_{t}^{N} f_{n}(x) \rightarrow \mathcal{P}_{t}^{N} f(x)
$$

for each $(t, x) \in \mathbb{R}_{+} \times K$, where $\mathcal{P}_{t}^{N} f_{n}$ and $\mathcal{P}_{t}^{N} f$ are the Neumann Poisson integrals of the functions $f_{n}$ and $f$, respectively. Moreover, the function

$$
u_{n}(t, x)=\mathcal{P}_{t}^{N} f_{n}(x)-u(t+1 / n, x)
$$

is harmonic and extends continuously to $\{0\} \times G_{n}$, because $f_{n}$ is continuous, with $u_{n}(0, x)=0$ for each $x \in G_{n}$. Moreover, $\left|u_{n}\right| \leq 2$ on the closure of $\Omega$, since $t+1 / n \leq 2$.

If we choose $v$ as in Lemma 5.3 , then

$$
\liminf _{(t, y) \rightarrow \partial \Omega}\left(2 v \pm u_{n}\right)(t, x) \geq 0
$$

so, by the maximum principle (Corollary 4.4), $2 v \pm u_{n} \geq 0$ on $\Omega$. Taking $n \rightarrow \infty$, we conclude that

$$
\left|\mathcal{P}_{t}^{N} f(x) \pm u\right| \leq 2 v
$$

on $\Omega$ and, as $\mathcal{P}_{t}^{N} f(x)$ and $v$ have nontangential limits (Theorem 3.10 and Lemma [5.3), we obtain Step 2.

Hence, for each $\alpha>0, u$ has a limit at the boundary, within the cone $\Gamma_{\alpha}(x)$, for every point $x$ in a subset $F_{\alpha}^{\prime} \subset F$ with $\mu\left(F \backslash F_{\alpha}^{\prime}\right)=0$.

If we take $F^{\prime}=\bigcup_{k} F_{k}^{\prime}$, we conclude that $u$ has nontangential limit at every point in $F^{\prime}$, with $\mu\left(E \backslash F^{\prime}\right)=0$, as desired.

\section{ACKNOWLEDGEMENTS}

The author would like to thank the referee for providing useful suggestions, which led to the improvement of some of the results of this paper.

\section{REFERENCES}

[ABR01] Sheldon Axler, Paul Bourdon, and Wade Ramey, Harmonic function theory, Springer, New York, N. Y., 2001, Graduate Texts in Mathematics, 137

[ASST03] Brian Adams, Alex S. Smith, Robert S. Strichartz, and Alexander Teplyaev, The spectrum of the laplacian on the pentagasket, Trends in Mathematics: Fractals in Graz 2001, Birkhäuser, Basel, 2003, pp. 1-24

[Bar98] Martin T. Barlow, Diffusions on fractals, Lectures on probability theory and statistics (Saint-Flour, 1995), Springer, Berlin, 1998, pp. 1-121

[CSW09] S. Constantin, R. S. Strichartz, and M. Wheeler, Analysis of the Laplacian and Spectral Operators on the Vicsek Set, ArXiv e-prints (2009), arXiv:0909.1066

[DSV99] Kyallee Dalrymple, Robert S. Strichartz, and Jade P. Vinson, Fractal differential equations on the sierpinski gasket, Journal of Fourier Analysis and Applications 5 (1999), 203-284

[FHK94] Pat J. Fitzsimmons, Ben M. Hambly, and Takashi Kumagai, Transition density estimates for Brownian motion on affine nested fractals, Commun. Math. Phys. 165 (1994), no. 3, 595-620

[IR10] M. Ionescu and L. Rogers, Complex Powers of the Laplacian on Affine Nested Fractals as Calderón-Zygmund operators, ArXiv e-prints (2010), arXiv:1002.2011

[Kig93] Jun Kigami, Harmonic calculus on p.c.f. self-similar sets, Trans. Amer. Math. Soc. 335 (1993), no. 2, 721-755

[Kig01] Jun Kigami, Analysis on fractals, Cambridge University Press, Cambridge, 2001 
[KL93] Jun Kigami and Michel L. Lapidus, Weyl's problem for the spectral distribution of Laplacians on p.c.f. self-similar fractals, Comm. Math. Phys. 158 (1993), no. 1, 93125

[Kum93] Takashi Kumagai, Regularity, closedness and spectral dimensions of the Dirichlet forms on P.C.F. self-similar sets, J. Math. Kyoto Univ. 33 (1993), no. 3, 765-786

[Lin90] Tom Lindstrom, Brownian motion on nested fractals, American Mathematical Society, Providence, RI, 1990

[ORS10] Kasso Okoudjou, Luke Rogers, and Robert Strichartz, Szegö limit theorems on the Sierpiǹski gasket, Journal of Fourier Analysis and Applications 16 (2010), 434-447

[Pel07] Anders Pelander, Solvability of differential equations on open subsets of the Sierpiǹski gasket, Journal d'Analyse Mathématique 102 (2007), 359-369

[RS78] Michael Reed and Barry Simon, Analysis of operators, Academic Press, New York, 1978, Methods of Modern Mathematical Physics, Volume 4

[RS10] Luke G. Rogers and Robert Strichartz, Distribution theory on fractals, Journal d'Analyse Mathématique (2010), to appear

[Sáe02] Ricardo A. Sáenz, Maximal functions related to the effective resistance metric on postcritically finite self-similar sets, Unpublished, 2002

[Ste70] Elias M. Stein, Singular integrals and differentiability properties of functions, Princeton University Press, Princeton, N.J., 1970, Princeton Mathematical Series, No. 30

[Str03] Robert S. Strichartz, Function spaces on fractals, Journal of Functional Analysis 198 (2003), no. $1,43-83$

[Str05] Robert Strichartz, Solvability for differential equations on fractals, Journal d'Analyse Mathématique 96 (2005), 247-267

[SW71] Elias M. Stein and Guido Weiss, Introduction to Fourier analysis on Euclidean spaces, Princeton University Press, Princeton, N.J., 1971, Princeton Mathematical Series, No. 32

Facultad de Ciencias, Universidad de Colima, Colima, Colima, Mexico, 28045

E-mail address: rasaenz@ucol.mx

$U R L:$ http://fejer.ucol.mx/ricardo 